\title{
Evaluation of peptide nanogels for accelerated wound healing in normal micropigs
}

\author{
Wafaa T Arab ${ }^{1}$, Abdullah M Niyas ${ }^{1,2}$, Kholoud Seferji ${ }^{1}$, Hepi H Susapto ${ }^{1}$, Irina Alexander ${ }^{1}$, Igor Cima ${ }^{1}$ and Charlotte AE Hauser ${ }^{*}$ \\ ${ }^{1}$ Laboratory for Nanomedicine, Division of Biological and Environmental Science and Engineering, King Abdullah \\ University of Science and Technology, Thuwal, Saudi Arabia. \\ ${ }^{2} \mathrm{Al}$ Faisal University College of Medicine, Riyadh, Saudi Arabia
}

\begin{abstract}
Non-healing chronic wounds are severe complications, which can often eventually lead to amputations. As such, there is a clear clinical need for dressings that promote the healing of chronic wounds. An advanced wound dressing aims to keep wound tissues moist while offering increased healing rates, preventing scar formation, reducing pain, minimizing infection, improving cosmetics, and lowering overall health care costs. We have previously developed tetrameric peptides AcIVZK- $\mathrm{NH}_{2}\left(\mathrm{Ac}-\mathrm{Ile}-\mathrm{Val}-\mathrm{Cha}-\mathrm{Lys}-\mathrm{NH}_{2}\right)$ and Ac-IVFK- $\mathrm{NH}_{2}\left(\mathrm{Ac}-\mathrm{Ile}-\mathrm{Val}-\mathrm{Phe}-\mathrm{Lys}-\mathrm{NH}_{2}\right)$ that self-assemble into nanofibrous hydrogels with biomimetic properties resembling those of collagen. In our study, we tested if these nanogels can fulfill the wound healing criteria mentioned above, and found that the nanogels are suitable scaffolds for encapsulating human dermal fibroblasts. We selected peptide nanogels Ac-IVZK-NH ${ }_{2}$ and $\mathrm{Ac}_{\mathrm{c}} \mathrm{IVFK}_{\mathrm{N}} \mathrm{NH}_{2}$ and produced silver nanoparticles in situ within the nanogels to assess their efficacy on micropigs with full-thickness excision wounds. The in situ generation of the silver nanoparticles was done solely through UV irradiation, no reducing agent was used. Application of the peptide nanogels on full thickness micropig wounds demonstrated that the scaffolds are biocompatible and did not trigger wound inflammation. This suggests that the scaffolds are safe for topical application. A comparison of the effect of both nanogels- even without the addition of the silver nanoparticles, revealed that the scaffold itself has a high potential to act as an antibacterial agent, which may suppress both the inflammatory reaction and the activity of proteases. Interestingly, the effect on wound closure of the peptide nanogels was comparable to those of standard care hydrogels. Despite our promising results, there is still much to learn about the molecular basis underlying the efficacy of tetrameric peptide nanogels in wound healing. This will support the urgent demand for advanced treatments of diabetic wounds, based on scientifically and clinically validated studies.
\end{abstract}

\section{Introduction}

An injury affecting the skin's protective barrier and function can be defined as a wound [1]. Skin injuries are further classified into two categories based on the nature of the restoration process. Acute wounds typically arise from tissue injuries after frictional contact between the skin and hard surfaces, and heal with minimal scarring within a short period of time [2]. Chronic wounds, on the other hand, result from repeated tissue injuries or underlying physiological conditions, taking at least 12 weeks for full healing such as in chronic diabetic ulcers and malignancies [3]. Wound healing is a complex energetic process consisting of four main steps: coagulation and hemostasis, inflammation, proliferation (granulation and contraction), and finally remodeling. In chronic wounds, the wound remains in the proliferation stage with excess inflammation and a failure to progress to remodeling [4]. The challenge of delayed wound healing has been the focus of many research studies. Existing literature provides a broad spectrum of treatment modalities striving to improve the healing process in chronic wounds. One method is the application of tissue engineering principles to facilitate the reestablishment of tissue integrity [5]. Bell and his group were the first to use composites with both dermal and epidermal components for treating patients with chronic burn wounds, but the results were not satisfactory [6]. They have also developed a matrix containing fibroblast/collagen known as Apligraft ${ }^{\bullet}$ [7].

Various dressings have been developed to avoid infection and enhance wound healing [8-10], such as collagen/polycaprolactone polymers [11] and sponges with bovine collagen I/chondroitin-4-6 and sulfate/chitosan [12]. Peptide nanogels offer a novel approach to enhance wound healing by improving existing synthetic materials, the majority of which have been polymers [1]. Peptide can have intrinsic antimicrobial and anti-inflammatory activity, which are crucial as the prolonged existence of infectious pathogens can result in a delayed healing process $[13,14]$. Peptides are biocompatible and offer tunable biodegradability $[13,14]$. Also, peptide nanogels combine the benefits of commercially available hydrogel dressings with those of nanofibrous scaffolds in providing the structural cues necessary for tissue restoration, in addition to providing an optical transparency that enables the observation of the wound. The soft properties of peptide nanogels reduce the pain that regularly occurs during routine wound dressing changes [1]. Different studies have investigated the use of hydrogel scaffolds as wound dressings and carriers of components that can promote healing. Studies on their effect on chronic wounds are scarce, however, and not satisfactory. The ideal dressing for chronic wounds should be free from contaminants and eliminate excess wound exudates, among

${ }^{\star}$ Correspondence to: Hauser CAE, Ph.D, Laboratory for Nanomedicine, Division of Biological and Environmental Science and Engineering, King Abdullah University of Science and Technology, Thuwal, Saudi Arabia, Tel: +966 12808 2524; Email: charlotte.hauser@kaust.edu.sa

Key words: ultrashort peptides, self-assembly, nanogels, chronic wounds, dressing, wound healing

Received: October 29, 2018; Accepted: November 16, 2018; Published: November 19, 2018 
other toxic components. Also, the dressing should maintain a moist environment, prevent the entrance of microorganisms, allow gaseous exchange, facilitate autolytic debridement, enhance granulation tissue formation, and provide cues to improve tissue regeneration [10]. In the past, nanofibrous silk materials [15] and different electrospun polymers [16] have been used as wound dressings, but, unlike hydrogel dressings, they failed to provide the moist environment necessary for cellular proliferation and debridement. Furthermore, natural and synthetic hydrogels made from alginate, chitosan, hyaluronic acid, and polyethylene glycol, do not produce nanofiber networks similar to the fibers found in the extracellular matrix (ECM) during their selfassembly processes, rather the resulting fibers are significantly larger, with dimensions on the microscale [17].

Our group focused on the development of novel synthetic peptidebased biomaterials $[18,19]$ that aim to combine the advantages of both natural and synthetic hydrogels. We have rationally designed and synthesized various different peptides with sequences consisting of three to seven amino acid residues. These peptides can self-assemble in water to form transparent nanofibrous hydrogels [20,21]. Among them, some amphiphilic ultrashort peptides have been shown to accelerate skin regeneration when used as dressings for burn wounds [1].

In this study, we have used previously designed tetrameric peptides that self-assemble into nanogels [22] to evaluate their efficacy in treating full thickness wounds in micropigs. The nanofibrous network structure was confirmed by scanning electron microscopy (SEM). Furthermore, in situ silver nanoparticle formation within the transparent hydrogels were examined by transmission electron microscopy (TEM). The biocompatibility of these nanofibrous hydrogels was tested on human dermal fibroblast (HDFn) to further assess the candidacy of these novel biomaterials for wound healing applications. Cell distribution, alignment, and phenotype were studied within a $3 \mathrm{D}$ culture environment by staining the actin cytoskeleton. We found that both peptide nanogels supported the proliferation of HDFn for up to three weeks and were thus deemed safe for topical application. To study the peptide nanogels in vivo, we topically applied them and their silver nanoparticle encapsulated counterparts to full thickness incision wounds in micropigs. We examined their healing capacity in comparison to commercially available standard of care hydrogel dressings, namely DuoDerm ${ }^{\star}$ Hydroactive ${ }^{\bullet}$ hydrogel and AQUACEL ${ }^{\circ}$ Ag EXTRATM-hydrofiberTM. The results showed a comparable effect when using these biomaterials both with and without silver nanoparticles (AgNPs) as well as the equivalent controls, thus demonstrating the biocompatibility of these materials and their ability to act as antibacterial agents. We propose that the peptide nanofibers were integrated into the wound area, thereby merging with existing ECM fibers and providing additional structural and functional support.

\section{Materials}

Two tetrameric self-assembling peptides IVFK (Ac-Ile-ValPhe-Lys- $\mathrm{NH}_{2}$ ) and IVZK (Ac-Ile-Val-Cha-Lys- $\mathrm{NH}_{2}$ ) were custom synthesized by Bachem AG, (Budendorf, Switzerland). Neonatal human dermal fibroblast cells (HDFn) were purchased from Gibco (Grand Island, USA). Dulbecco's Modified Eagle Medium (DMEM), fetal bovine serum (FBS), Dulbecco's phosphate-buffered saline (PBS) solution, and penicillin-streptomycin antibiotics $(\mathrm{P} / \mathrm{S})$ were purchased from Gibco (Grand Island, USA). CellTiter- Glo ${ }^{\circ}$ Luminescent 3D cell viability assay kits were ordered from Promega (Madison, USA). MTT Cell Proliferation Assay kit and LIVE/DEAD ${ }^{\circledR}$ Viability/ Cytotoxicity kit were purchased from either ThermoFisher Scientific,
USA, Promega, USA or Life TechnologiesTM, USA. Immunostaining antibodies antivinculin and Rhodamine-Phalloidin were purchased from Invitrogen, USA, and anti-mouse IgG-FITC was purchased from Sigma, USA. T175 or T75 cell culture flasks and 96-well plates were ordered from Corning, USA.

\section{Methods}

\section{Peptides hydrogel preparation}

IVZK and IVFK peptide powders were dissolved in Milli-Q water Then, 10x phosphate buffered saline was added to the peptide solution. Gelation of both peptides occurred within a few seconds at minimum concentration of $4 \mathrm{mg} / \mathrm{mL}$ and $3 \mathrm{mg} / \mathrm{mL}$ for IVFK and IVZK, respectively. The final volume ratio of peptide solution and 10x PBS was 9:1.

\section{Evaluation of fiber structures by field-emission scanning microscopy (FE-SEM)}

The peptide nanogels were dehydrated by gradually increasing concentrations of $30 \%, 50 \%, 70 \%, 90 \%$ and $100 \%$ (v/v) ethanol solutions for $15 \mathrm{mins}$ in each solution.. Further dehydration in $100 \%$ ethanol solution was continued by changing the absolute ethanol solution with a fresh one twice for 15 mins each. The dehydrated samples were subsequently kept in 1:2 (v/v) hexamethyldisilazane (HMDS): ethanol for $20 \mathrm{~min}$, followed by 20 mins of incubation in a fresh solution of 2:1 HMDS: ethanol and then two times of 20 mins in 100\% HMDS. Finally,the samples were kept inside a fume hood overnight to allow HMDS to evaporate. Prior to imaging, the samples were mounted onto SEM stubs using conductive carbon tape, and then sputter-coated with a 5 $\mathrm{nm}$ thick coating of Iridium and a $3 \mathrm{~nm}$ thick coating of Gold/Palladium. The coated samples were then imaged with a Field Emission Scanning Electron Microscopy system (FEI Nova Nano630 SEM, Oregon, USA).

\section{Evaluation of silver nanoparticles (AgNPs) formation by transmission electron microscopy (TEM)}

Silver nanoparticle studies were carried out as previously described [23]. Briefly, the peptide powders were dissolved in distilled water. Then, $100 \mathrm{mM}$ of silver nitrate (AgNO3) in Tris buffer $(\mathrm{pH}=8.5)$ was added into the peptide solution for a final concentration of $10 \mathrm{mM}$. Gelation occurred within 5 minutes at the minimum concentration of 4 $\mathrm{mg} / \mathrm{mL}$ IVFK and $3 \mathrm{mg} / \mathrm{mL}$ IVZK. The gels were then exposed to 254 nm UV light for 5 minutes allowing for in situ formation of AgNPs. A small portion of the gel was taken and diluted in water. Then, 10ul of this diluted gel was dropped onto carbon-coated cupper grid and kept there for 5 min before blotting with filter paper. The grid was then dried overnight at RT. TEM analysis was performed with the Tecnai G2 Spirit Twin instrument with an accelerating voltage of $120 \mathrm{kV}$ using an emission gun.

\section{Rheology studies}

The viscoelastic properties of peptide nanogels were characterized by using the TA Ares-G2 rheometer with an $8 \mathrm{~mm}$ parallel-plate geometry. The peptide gels were prepared in a $8 \mathrm{~mm}$ diameter polymethylmethacrylate ring, which were later being incubated inside a sealed tissue-culture dish at $40^{\circ} \mathrm{C}$ for overnight before the measurements. For each peptide, six replicates of gels having a volume of $150 \mu \mathrm{L}$ were prepared. The measurements were conducted at a 1.5 $\mathrm{mm}$ gap distance with a temperature of $40^{\circ} \mathrm{C}$ to mimic conditions used for cell growth. Three consecutive tests, such as time-sweep, frequencysweep, and amplitude-sweep, were carried out for each sample to confirm the mechanical stiffness of the gels. Time-sweep analysis was 
performed for 5 minutes at an angular frequency of $1 \mathrm{~Hz}$ and at 0.1 $\%$ strain to observe storage and loss modulus at an early stage, before the next measurements were done. A frequency sweep analysis was subsequently carried out by reducing the angular frequency from 100 to $0.1 \mathrm{~Hz}$ while keeping the strain at $0.1 \%$. Lastly, amplitude sweep analysis was performed to observe the minimum value of the amplitude strain needed to break the gel structure. This test was performed with an angular frequency and strain of $1 \mathrm{~Hz}$ and $0.01 \%-100 \%$, respectively.

\section{Cell Culture}

Human dermal fibroblast (HDFn) were cultured either in T175 or T75 culture flasks in complete DMEM media (10\% fetal bovine serum, and $1 \%$ penicillin/ streptomycin). The cells were incubated in a humidified incubator with $95 \%$ air and $5 \% \mathrm{CO} 2$ at $37^{\circ} \mathrm{C}$. The cells were subcultured using trypsin at approximately $80 \%$ confluence. The culture media was replenished every $48 \mathrm{~h}$.

\section{Establishing 3D culture}

$3 \mathrm{D}$ cell culture was performed as previously described [22,24]. Briefly, a gel base at the bottom of a cell culture plate was created by pipetting a peptide solution (peptide with water) into a glass bottom dish (Nunc, $12 \mathrm{~mm}$ ) and mixing it with 2x PBS. After gel formation, the peptide solution was pipetted on top of this gel base and mixed with human dermal fibroblasts (25,000 cells/dish) suspended in 2x PBS. Gelation occurred within 3-5 minutes. Then, the culture medium was subsequently added on top of each construct. The plate was incubated at $37^{\circ} \mathrm{C}, 95 \%$ air and $5 \% \mathrm{CO}_{2}$. Different biocompatibility assays were applied using this construct.

\section{Biocompatibility evaluations of peptide nanogels}

All the biocompatibility studies, including cell viability assay (MTT assay), ATP production (3D cell proliferation assay), and live/ dead assay, as well as morphological changes in response to peptide nanogels, were performed as previously described [22,24].

\section{In vivo wound healing evaluation by peptide nanogels in micropigs}

The study was designed as a case-control study. All experimental protocols and animal care complied with the "Guide for the care and use of Laboratory Animals" (PWG, 15 Tech Park Cres, Singapore 638117; Prof Lim Thiam Chye, Department of Surgery, Yong Loo Lin Medical School, National University of Singapore).

\section{Animals and maintenance}

A total number of 10 healthy micropigs (Sus Scrofa), (5 male and 5 female PWG micropigs) of comparable age (adult, over 16 months), with a weight of approximately $25-30 \mathrm{~kg}$ at the start of the experiment, were selected for the wound healing study. Before initiating the experiment, the animals were acclimatized for 3-7 days. During this period, all animals were daily observed for any clinical signs of disease. A unique number associated with the ear-tag number identified each animal. Then, all pens were labeled with a cage card indicating study number, and animal ID. The cages equipped with food bowls and water bottles. The room temperature was $22-30^{\circ} \mathrm{C}$ with $50-80 \%$ humidity and approximately 12 hours of 150-300 lux of the light/dark cycle. Each animal was fasted overnight (at least 12 hours) before the experimental procedures and deprived of food during wound creation and dressing. After wound creation, each animal was individually housed, using steel cages with a size of approximately $80 \mathrm{~cm} \times 140 \mathrm{~cm} \times 75 \mathrm{~cm}$ and defined by a marked cage card.

\section{Wounding and chamber treatment}

For all surgical procedures, intramuscular Ketamine eats $10 \mathrm{mg} /$ $\mathrm{kg}$, Atropine at $0.05-0.10 \mathrm{mg} / \mathrm{kg}$ and Xylazine eats $2-5 \mathrm{mg} / \mathrm{kg}$ intramuscularly was administered at the time of anesthetic induction. General anesthesia was maintained with $2-5 \%$ isoflurane in oxygen. The anesthetized pigs were placed in stern recumbency and the hair removed, as required. Care was taken to avoid mechanical irritation and trauma. The surgical site was prepared by cleaning and sterilizing with iodine followed by $70 \%$ ethanol. A sterile surgical drape was used to drape the surgical site.

\section{Full-thickness wound creation}

In total 16 wounds ( $2 \mathrm{~cm}$ in diameter) were introduced on the back of each pig via surgical incision down to muscular layer. The incisions in a $4 \times 4$ pattern were approximately $4 \mathrm{~cm}$ apart of each other on either side of the dorsal back of the animal.

\section{Hydrogels as wound dressings for micropigs full thickness wound}

Two different ultrashort peptide nanogels IVFK and IVZK and their variations that contained silver nanoparticles, IVFK-AgNPs and IVZK-AgNPs, were chosen in this study. Standard of care hydrogelbased dressings known as Duoderm Hydroactive $\mathrm{Gel}^{\circ}$ and Aquacel ${ }^{\circ}$ Hydrofiber AG, were chosen as controls. All dressings were topically applied and were slightly extended over the wound by approximately 1 $\mathrm{cm}$. This guaranteed full coverage of the entire wound in a randomized pattern. Then, waterproof adhesive films, Tegaderm (3M Singapore), were used to secure the dressings with further protected by a covering. Animals were housed individually to avoid any disturbances to the wounds potentially caused by other pen mates. Wound dressings changes were done twice a week.

\section{Wound healing assessment}

Wound contraction and wound closure were evaluated using digital planimetry. The tattooed margins were checked, on days $4,8,11,15,18$, 22 , and 25 after initiation of the study. A photograph of the wound was taken every time wound change was conducted using amount at a fixed distance above the wound and a ruler as a scale adjacent to the wound and analyzed the data using image J software. The general linear model for the determination of time versus wound closure (re-epithelialization) and granulation tissue formation regarding each treatment was evaluated. The percentage of wound closure was considered by the formula: Wound closure $(\%)=\frac{\text { area at biopsy }}{\text { the area on incision day }} \times 100$

and the percentage of wound area was calculated by applying the following equation:

$$
\text { Wound area }(\%)=\frac{(\text { wound area at day } 0-\text { wound is at day } x)}{\text { wound area at day } 0} \times 100
$$

\section{Terminal procedure (animal euthanasia)}

The animals were anesthetized with isoflurane. They were sacrificed by exsanguination of the vena cava and aorta.

\section{Statistical analysis}

Three independent experiments were done for each type of test. A one-way analysis of the variance was used to determine the statistical differences between the experimental groups. Regarding the wound closure and re-epithelialization data, the wound area over observation time was considered. All results are presented as a mean \pm SD. Results 
were considered to be statistically significant when the p-values were $\mathrm{P}<0.05$.

\section{Results and discussion}

Impairment of wound healing in patients with complicated chronic wounds as well as in patients with diabetic ulcers affects the quality of life of millions of people and requires a high cost to cure. Wound healing is a complicated process that relies on communication between different cell types, growth factors, and extracellular matrix molecules. Various dressings have been developed to enhance wound healing in individuals without co-morbidities, typically based on enhancing debridement, cleaning and offering a moist environment conducive to healing.

Herein, we evaluated the efficiency of previously designed tetrameic ultrashort aliphatic peptide hydrogels (IVFK and IVZK) [22] as primary dressings for full thickness wounds. These peptides have a native ability to self-assemble into fibrous scaffolds in aqueous solution. SEM confirmed a dense nanofibrous network formation (Figure 1A and $1 \mathrm{~B}$ ) where the fibers structurally resemble collagen fibers with respect to topography [25]. This suggests their use as scaffolds for skin regeneration [26-29], particularly for full thickness wounds. The diameter of these nano-scale fibers ranges within the diametric scope found in the natural ECM $(5-300 \mathrm{~nm})$ [30]. This distinctive fibrous structure allows the gel to entrap $>99 \%$ water within its bulk volume. It preserves a moist environment and leaves the tissue hydrated which in turn reduces the pain during frequent dressing changes [1]. Additional advantages of the peptide nanogels are their optical transparency that allows for observation of the wound. Another advantage of selecting these peptides is that the self-assembly can be enhanced in the presence of phosphate buffer saline (PBS), which usually is used to prepare wound dressings. This preserves the physiological $\mathrm{pH}$ and enhances the wound dressing capacity to absorb wound exudates. Importantly, the short length of the peptides offers the advantage of a low cost production different to other protein- or polypeptide-based hydrogels. Also, these nanogels comprise a polar lysine amino acid residue, which aids the wound homeostasis [31]. In addition, the porous nanofibrous structure supports the in situ fabrication of silver nanoparticles (AgNPs) from a silver nitrate solution without using any chemical reducing agent (Figure 1C and 1D). TEM confirmed monodispersed AgNPs with a size range of $10-20 \mathrm{~nm}$, which has been verified as a sufficient size to elicit antibacterial activity [32].

The mechanical stiffness and stability of both peptide nanogels were determined using oscillatory rheology that is based on measuring the storage modulus (G') and loss modulus (G”). Samples were examined through time-sweep analysis in a linear viscoelastic range (LVR) for five minutes by keeping the storage modulus constant under elastic deformation. The G' values of IVFK and IVZK were found to be around $18.9 \mathrm{kPa}$ and $56.1 \mathrm{kPa}$, respectively, while their G" values were less than an order of magnitude from their $G$ ' indicating the gel state of both samples, as shown in figure 2A [33]. Both samples also showed a frequency-independent behavior, without any crossover at lower frequencies, which is a common viscoelastic property for hydrogel (Figure 2B) [34]. An amplitude sweep test demonstrated the length of the LVR under increasing strain values. It was found that the LVR for both peptide gels was still constant under $0.2 \%$ strain. However, the later cross-over point of IVFK where G' = G' indicated that IVFK could tolerate higher strain before the breaking of the gels, in addition to its high G' value (Figure 2C) [35].

As cellular proliferation, adhesion and the formation of threedimensional cellular networks are extremely important for tissue
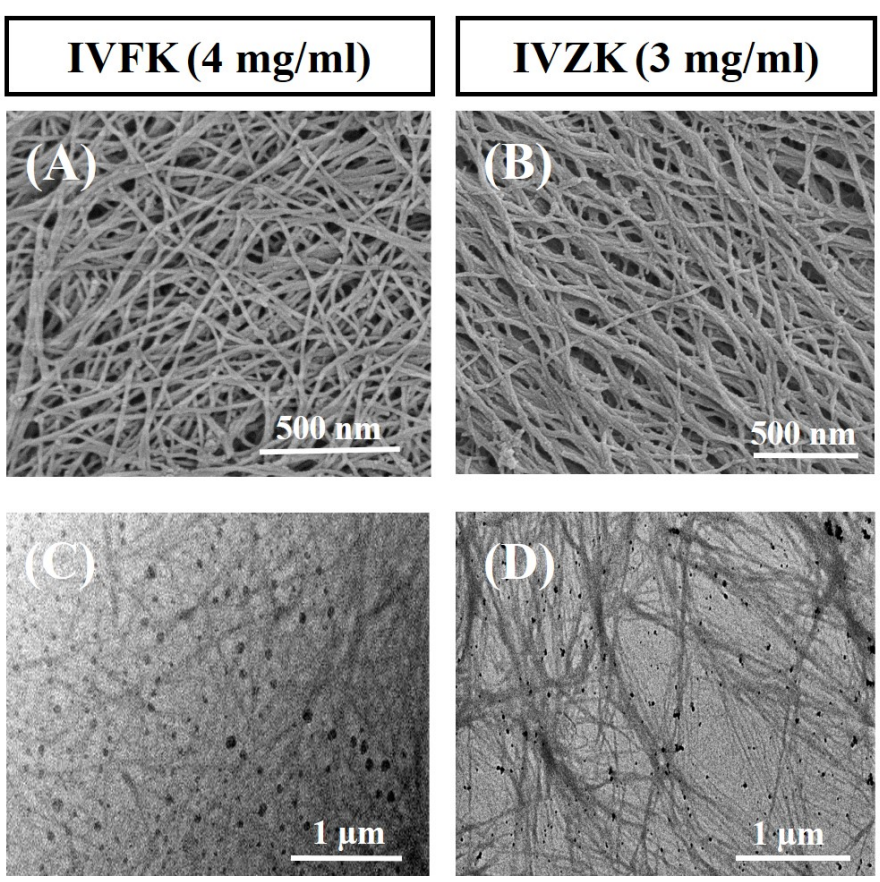

Figure 1. Ultrashort peptides self-assemble into three-dimensional nanofibrous networks. Field emission scanning electron microscopy images of $4 \mathrm{mg} / \mathrm{ml}$ IVFK (A) and $3 \mathrm{mg} / \mathrm{ml}$ IVZK (B). Transmission electron microscopy images of silver nanoparticles formation in 4 $\mathrm{mg} / \mathrm{ml}$ IVFK (C) and $3 \mathrm{mg} / \mathrm{ml} \mathrm{IVZK} \mathrm{(D)} \mathrm{in} \mathrm{water} \mathrm{using} 10 \mathrm{mM} \mathrm{AgNO}_{3}$ in Tris buffer at $\mathrm{pH} 8$

repair and regeneration, cytocompatibility of the peptide nanogels were evaluated using neonatal human dermal fibroblast cells. The in vitro investigation demonstrated that exposure of HDFn to different concentrations of peptide nanogels did not affect cell growth when compared to cell growth in tissue culture plates (TCP), as shown in figure 3. Further, a time-dependent increase in ATP production was observed in 3D cultured HDFn for 7, 14, and 21 days (Figure 4), demonstrating that the encapsulated human dermal fibroblasts were metabolically active up to three weeks. Moreover, the fluorescence intensity of green color resulting from the cytotoxicity assay revealed that the majority of cells were alive up to 21 days, with very few dead cells (Figure 5). Furthermore, fluorescence confocal z-stack images of the actin cytoskeleton, which gives direct evidence for cellular morphology and cytoskeleton structure [36], demonstrated that after a period of seven days, cells proliferated and extended within the scaffolds (Figure 6A-6C). At day 14, the fibroblast growth rapidly increased and created a network via cell-to-cell junctions (Figure 6D$6 \mathrm{~F})$. Continuous growth of encapsulated cells and the formation of an extensive network saturating the nanogel matrix, was observed at day 21 (Figure 6G-6I). Upon this observation, we could confirm that the peptide scaffolds provided a favorable microenvironment for cell adhesion, spreading and proliferation. Therefore, we hypothesized that the compatible peptide biomaterials might have an ability to boost the healing of chronic wounds and enhance tissue regeneration.

For our in vivo wound healing study, micropigs were used as a suitable animal model, because pigs, although a costly animal model, resemble humans regarding skin structure and wound healing characteristics [37]. Sullivan, et al. have been considered pigs as a satisfying animal model for research related to skin, when comparing the outcomes obtained on clinical studies, and the results obtained on pigs, small mammals, as well as within in vitro studies [38]. Further, the pre-clinical porcine model has efficiently been used to assess the safety and efficacy of peptide nanogels for wound healing, due to its 

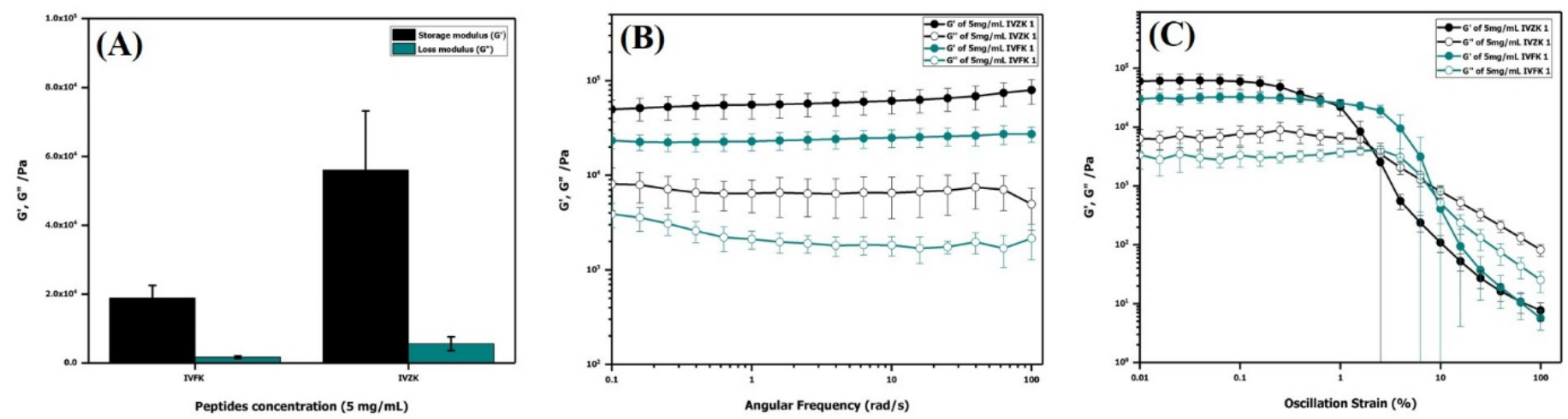

Figure 2. Rheology result of $5 \mathrm{mg} / \mathrm{ml}$ IVFK and IVZK peptide nanogels at a temperature of $40^{\circ} \mathrm{C}$. (A). Equilibrium moduli at 5 minutes, (B) oscillatory frequency sweep test, and (C) oscillatory amplitude sweep test
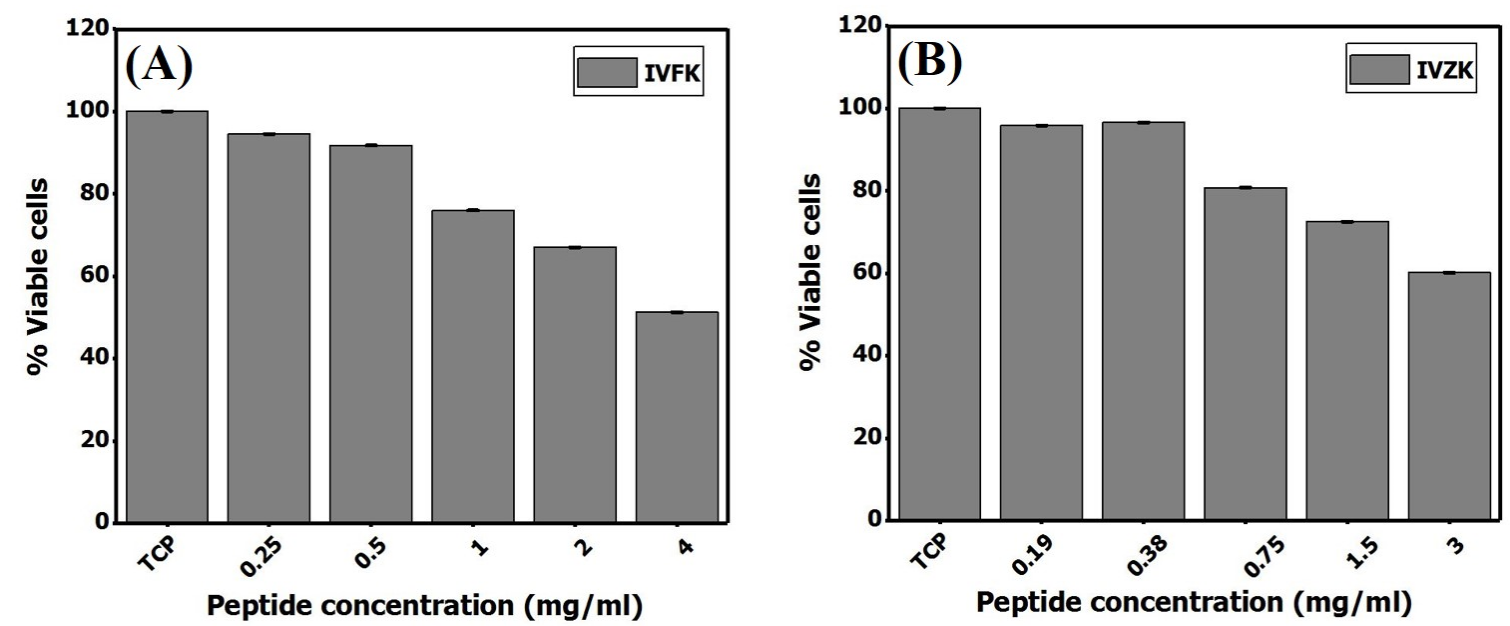

Figure 3. Graphical representation of an MTT assay using human dermal fibroblast cells in the presence of different peptide concentrations within a period of 24 hours. IVFK (A) and IVZK (B)

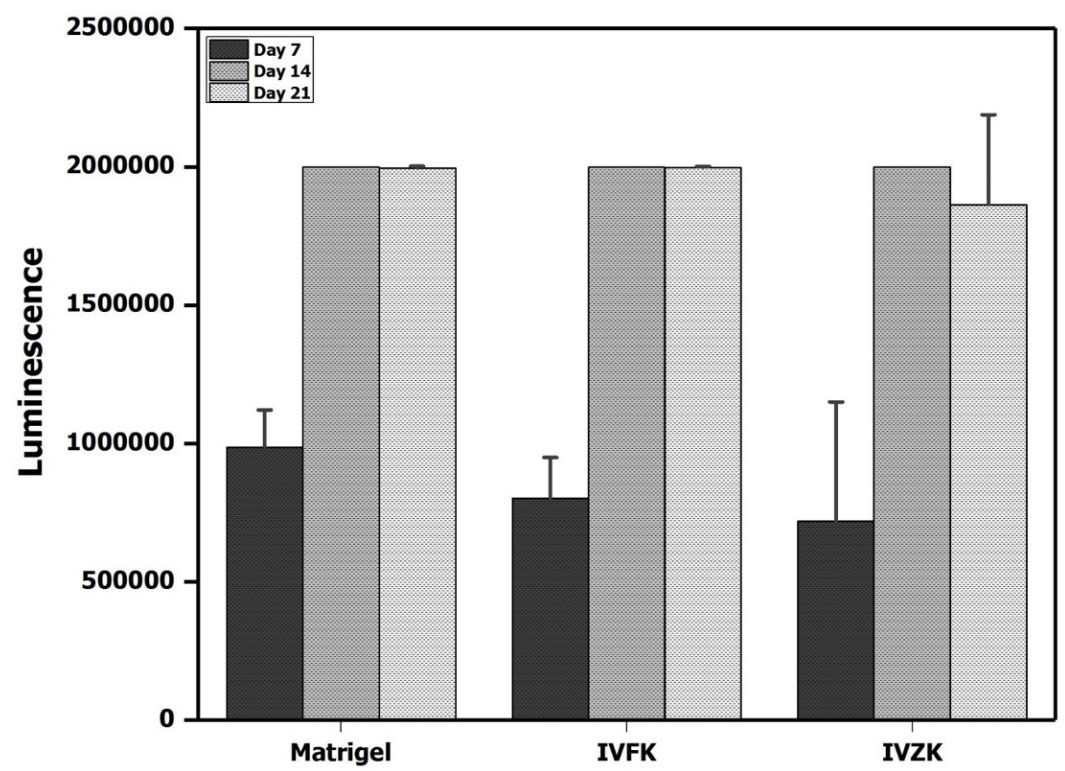

Figure 4. 3D viability assay of human dermal fibroblast cells encapsulated in peptide nanogels, $4 \mathrm{mg} / \mathrm{ml} \mathrm{IVFK}$ and $3 \mathrm{mg} / \mathrm{ml}$ and $4 \mathrm{mg} / \mathrm{ml}$ Matrigel at different time points, such as at $3,7,14$ and 21 days 


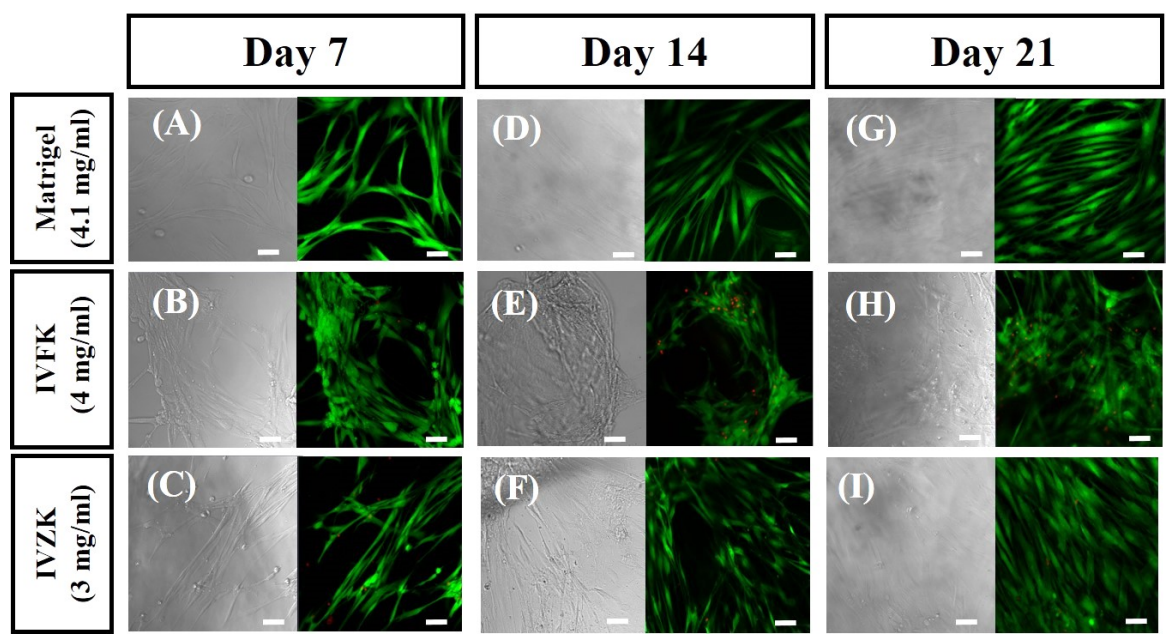

Figure 5. Cytotoxicity results of human dermal fibroblast cells encapsulated in peptide nanogels, $4 \mathrm{mg} / \mathrm{ml} \mathrm{IVFK}$ and $3 \mathrm{mg} / \mathrm{ml} \mathrm{IVZK}$, and $4 \mathrm{mg} / \mathrm{ml}$ Matrigel, at different time points. Matrigel was used as a positive control (A, D, G), IVFK (B, E, H), and IVZK (C, F, H) at days 7, 14 and 21, respectively. Scale bars $50 \mu \mathrm{m}$

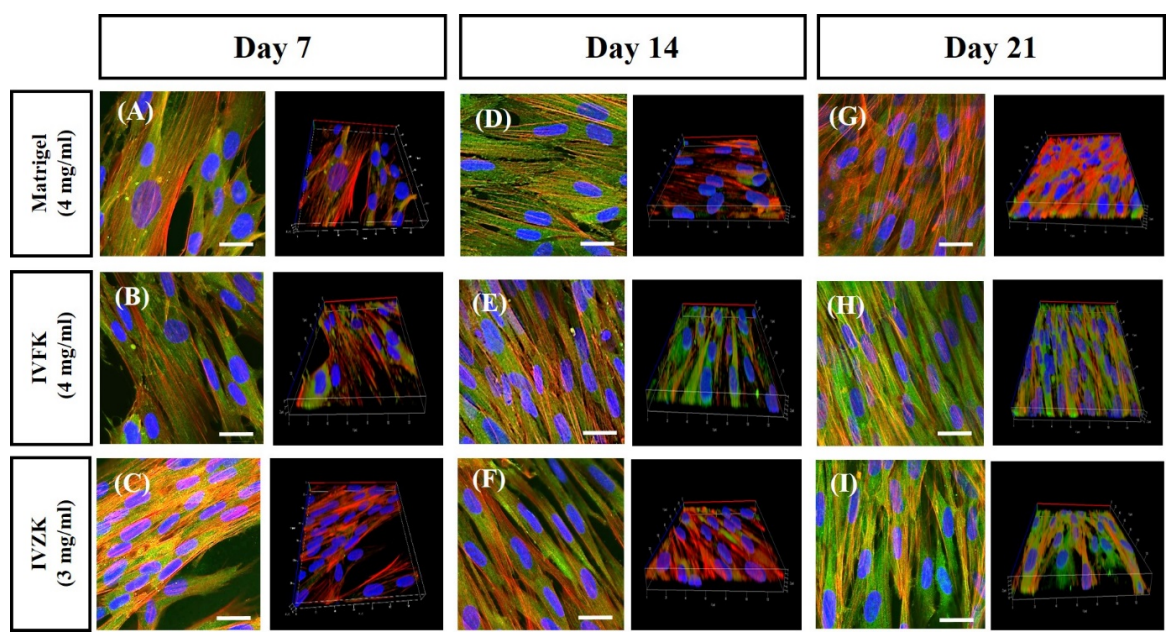

Figure 6. Overlaid Z-stack confocal fluorescence images of human dermal fibroblast cells encapsulated in peptide nanogels (4mg/ml IVFK and 3mg/ml IVZK) and control Matrigel (4mg/ $\mathrm{ml}$ ) at different time points. Nucleus is shown in blue, F-actin is shown in red and vinculin in green. Matrigel (A, D, G), IVFK (B, E, H), and IVZK (C, F, I) nanogels at day 7, 14, and 21, respectively. Scale bar is $20 \mu \mathrm{m}$

anatomical and functional similarity to humans when compared to other animal models [39]. Applying 25 different wound therapies using porcine models and comparing it with studies on humans revealed that the data of $78 \%$ of the porcine studies agreed with those of the human studies, whereas only $53-57 \%$ showed agreement when using small-mammal models [38].

Full-thickness incision wounds $(1 \times 1 \mathrm{~cm})$ were created on the dorsal back of all micropigs by surgical removal of the upper epidermis layer and middle dermis layer. The newly created wounds were used to evaluate the therapeutic effects of different biomaterials on the wound closure. A block of different hydrogel dressing (IVFK, IVZK, IVFKAgNPs, IVZK-AgNPs) or DuoDerm ${ }^{\star}$ and Aquacel $^{\circledR}$ (positive controls) was topically applied. Then, Tegaderm was used to protect and cover the wounds and to allow gaseous exchange.

After each wound dressing change, digital planimetry was applied to quantify the re-epithelialization and granulation tissue formation. Re-epithelialization is very important for protecting the host against pathogens as the prolonged exposure to pathogens can cause excessive inflammation that affects the organ functions and delays or undermines the skin regeneration process [1]. Granulation tissue, on the other hand, known to occur with proliferating fibroblast and formation of newly microvasculature was observed in some pigs in their open wounds at day 8 , with a percentage of $26.7 \%, 28.8 \%, 24.5 \%, 23.4 \% 33.8 \%$ and $27.8 \%$ for duoderm, aquacel, IVFK, IVZK, IVFK- AgNPs and IVZKAgNPs, respectively (Figure 7D). The granulation tissue formation was denser in the IVFK-AgNO3 treated group compared to the controls and other treated hydrogels groups. However, the difference did not reach statistical significance. After 11 days of treatment, enhanced re-epithelialization and formation of granulation tissue at the wound edge, when compared to the center of the wound, was observed in all studied micropigs, as shown in figure 7C and D. Nearly complete re-epithelialization was achieved at day 22 with a gradual reduction in granulation tissue formation. The presence of granulation tissue suggests that both hydrogels support the proliferation of endothelial cells and thus enhance the angiogenesis.

Statistical analysis revealed that after four days of treatment the percentage of the wound area was slightly reduced by IVFK hydrogels (79.2\%) and IVZK hydrogels (79\%), compared with duoderm (83\%), aquacel (81\%), IVFK- AgNPs (91.7\%) and IVZK- AgNPs (81.4\%). However, the reduction was not significant (Figure 7 A, B). Chronic 

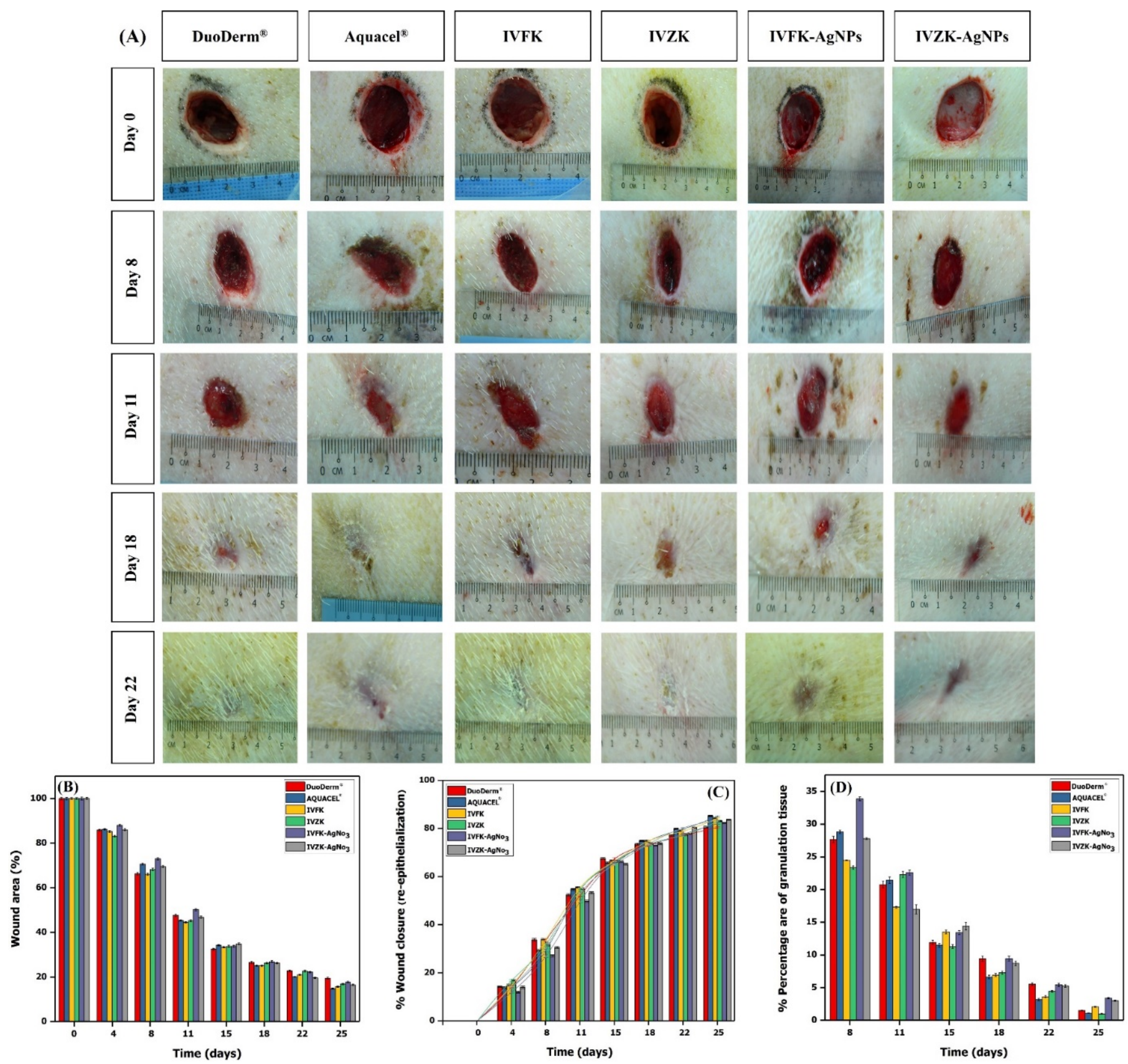

Figure 7. Representative pictures of wound contraction after application of different peptide nanogels in comparison to standard care (A). Quantitative evaluation of wound area contraction in response to different treatments (B). The percentage of re-epithelization (C) and granulation tissue formation reduction over the time (D) is shown, using digital planimetry. The error bars denote the standard error of the mean $(n=10)$

wounds are characterized by a prolonged inflammatory reaction, which results in imbalances in macrophage functions leading to a significant decrease in the pro-healing macrophage phenotype, when compared to the pro-inflammatory macrophage [40] which results in increased protease secretion at the wound site [41,42]. Silver-based antibiotics was used to prevent the infection of the wound $[43,44]$. It is worth mentioning that both peptide nanogels with and without silver nanoparticles had a similar effect on wound closure, demonstrating that these hydrogels have intrinsic antimicrobial activity comparable to the silver nanoparticles that suppress the inflammatory response and inhibit the activity of the protease. Also, there were no visually apparent differences among all tested conditions, including both controls, peptide nanogels with and without silver nanoparticles in respect to minimal scar formation and negligible infection.

The application of these hydrogels to chronic full-thickness wounds stimulate granulation tissue formation as well as the reepithelialization which eventually close the wound without the addition of exogenous growth factors or cells [45]. Also, the observed ability of these peptide nanogels alone, without any additional additive factors, to exhibit a similar effect as the controls will enable future development of 3D scaffolds containing skin/stem cell as well as angiogenic factors to promote vascularization that is necessary for successful tissue engineering graft. Moreover, these peptide nanogels, containing only 
four amino acids, are less costly and devoid of any chemical additive, in contrast to commercially available hydrogel dressings. Considering the results we believe that the utilized peptide nanogels are promising materials for fabricating skin substitutes as well as 3D skin graft models, particularly in the context of wound healing.

This study is giving preliminary in vitro and in vivo evidence on the efficacy of the investigated peptide nanogels as promising wound dressings. Further studies are necessary and mandatory to check closely and in more detail on wound hydration, autolytic debridement, inflammation, cytokine expression, and metalloproteinases activity.

\section{Conclusion}

Our study shows that newly developed peptide nanogels provide native cues to human dermal fibroblast cells and promote their proliferation as well as their extensive network formation in vitro. The application of our ultrashort peptide nanogels on full thickness wounds in a minipig model demonstrated biocompatibility with micropig skin tissue. The peptide nanogels did not trigger wound inflammation and thus can be considered to be safe for topical applications. The peptide-based biomaterials exhibited a similar effect in wound closure when compared to the current standard of care hydrogels. Moreover, the comparable effect obtained after using hydrogels with/without AgNPs indicated that these materials have a high potential to act as an antibacterial agent. Our results suggest that peptide nanogels as a wound dressing have the prospect to strongly enhance chronic wound healing when combining with cells or growth factors that are suitable for skin tissue regeneration. We propose that the peptide nanogels could act as potential carriers for HDFn transplantation in in vivo therapies and as promising biomaterials for tissue engineering applications. Further in vivo studies should be performed to assess how the 3D culture peptide scaffolds work when seeded together with autologous skin cells. Follow-up studies are critically needed as they will allow for a more precise evaluation of the dressings' fate post- grafting.

\section{Acknowledgment}

We want to thank Dr. Sakandar Rauf and Mr. Fares Al-Toub for their help in the preparation of the in vivo wound healing study. Also, we would like to thank Mr. Abdul Aziz Al-Kibsi, Ms. Leenh BaHammam and Ms. Enjey Gazzawi for their help in analyzing the resulting data to assess rates of wound closure by digital planimetry. We want also to thank Ms. Sarah Ghalayini for kindly proof-reading the manuscript. The research reported in this publication was supported by funding from King Abdullah University of Science and Technology (KAUST).

\section{Author Contributions}

C.A.E.H designed and supervised the project. W.A and C.A.E.H wrote the manuscript. W.A, A.N, K.S and H.S performed the majority of the experiments. I.A. and I.C. helped with the preparation and set-up of the study.

\section{Conflict of Interest and Funding}

The authors declare that they do not have any competing interests.

\section{References}

1. Loo Y, Wong YC, Cai EZ, Ang CH, Raju A, et al. (2014) Ultrashort peptide nanofibrous hydrogels for the acceleration of healing of burn wounds. Biomaterials 35: 4805-4814.

2. Pham C, Greenwood J, Cleland H, Woodruff P, Maddern G (2007) Bioengineered skin substitutes for the management of burns: a systematic review. Burns 33: 946-957. [Crossref]
3. CFD Center for Disease Control and Prevention (2014) National diabetes statistics report: estimates of diabetes and its burden in the United States, Atlanta, GA: US Department of Health and Human Services 2014

4. Fox LT, Mazumder A, Dwivedi A, Gerber M, du Plessis J (2017) In vitro wound healing and cytotoxic activity of the gel and whole-leaf materials from selected aloe species. $J$ Ethnopharmacol 200: 1-7. [Crossref]

5. Langer R, Vacanti JP (1993) Tissue engineering. Science 260: 920-926. [Crossref]

6. Wassermann D, Schlotterer M, Toulon A, Cazalet C, Marien M, et al. (1988) Preliminary clinical studies of a biological skin equivalent in burned patients. Burns 14: 326-330.

7. Eaglstein WH, Falanga V (1997) Tissue engineering and the development of Apligraf, a human skin equivalent. Clin Ther 19: 894-905. [Crossref]

8. Rogers LC, Bevilacqua NJ, Armstrong DG, Andros G (2010) Digital planimetry results in more accurate wound measurements: a comparison to standard ruler measurements, SAGE Publications.

9. Gao M, Nguyen TT, Suckow MA, Wolter WR, Gooyit M, et al. (2015) Acceleration of diabetic wound healing using a novel protease-anti-protease combination therapy. Proceedings of the National Academy of Sciences 112: 15226-15231.

10. Snoek-van beurden PA, Von den Hoff JW (2005) Zymographic techniques for the analysis of matrix metalloproteinases and their inhibitors. Biotechniques 38: 73-83.

11. Dai NT, Williamson MR, Khammo N, Adams EF, Coombes AG (2004) Composite cell support membranes based on collagen and polycaprolactone for tissue engineering of skin. Biomaterials 25: 4263-4271.

12. Li H, Berthod F, Xu W, Damour O, Germain L (1997) Use of in vitro reconstructed skin to cover skin flap donor site. $J$ Surg Res 73: 143-148. [Crossref]

13. Wolcott R, Sanford N, Gabrilska R, Oates JL, Wilkinson JE (2016) Microbiota is a primary cause of pathogenesis of chronic wounds. J Wound Care 25: S33-S43. [Crossref]

14. McCloskey AP, Gilmore BF, Laverty G (2014) Evolution of antimicrobial peptides to self-assembled peptides for biomaterial applications. Pathogens 3: 791-821.

15. Gil ES, Panilaitis B, Bellas E, Kaplan DL (2013) Functionalized silk biomaterials for wound healing. Adv Healthc Mater 2: 206-217.

16. Choi JS, Leong KW, Yoo HS (2008) In vivo wound healing of diabetic ulcers using electrospun nanofibers immobilized with human epidermal growth factor (EGF). Biomaterials 29: 587-596.

17. Rice JJ, Martino MM, De Laporte L, Tortelli F, Briquez PS, et al. (2013) Engineering the regenerative microenvironment with biomaterials. Adv Healthc Mater 2: 57-71.

18. Seow WY, Hauser CA (2014) Short to ultrashort peptide nanogels for biomedical uses. Mater Today 17: 381-388.

19. Wu EC, Zhang S, Hauser CA (2012) Self-assembling peptides as cell-interactive scaffolds. Adv Functional Mater 22: 456-468.

20. Hauser CAE, Deng R, Mishra A, Loo Y, Khoe U, et al. (2011) Natural tri-to hexapeptides self-assemble in water to amyloid $B$-type fiber aggregates by unexpected a-helical intermediate structures. Proceedings of the National Academy of Sciences 108: $1361-1366$

21. Mishra A, Loo Y, Deng R, Chuah YJ, Hee HT, JY Ying, et al. (2011) Ultrasmall natural peptides self-assemble to strong temperature-resistant helical fibers in scaffolds suitable for tissue engineering. Nano Today 6: 232-239.

22. Rauf S, Kahin K, Alshehri S, Abdelrahman S, Susapto HH, et al. (2018) In situ $3 \mathrm{D}$ bioprinting under physiological $\mathrm{pH}$ using ultrashort self-assembling peptides. (submitted).

23. Reithofer MR, Lakshmanan A, Ping AT, Chin JM, Hauser CAE (2014) In situ synthesis of size-controlled, stable silver nanoparticles within ultrashort peptide nanogels and their anti-bacterial properties. Biomaterials 35: 7535-7542.

24. Arab W, Rauf S, Al-Harbi O, Hauser CAE (2018) Novel Ultrashort Self-Assembling Peptide Bioinks for 3D Culture of Muscle Myoblast Cells. Int J Bioprinting 4: 1-13.

25. Loo Y, Lakshmanan A, Ni M, Toh LL, Wang S, et al. (2015) Peptide bioink: selfassembling nanofibrous scaffolds for three-dimensional organotypic cultures. Nano Letters 15: 6919-6925.

26. Ellis-Behnke RG, Liang YX, You SW, Tay DK, Zhang S, et al. (2006) Nano neuro knitting: Peptide nanofiber scaffold for brain repair and axon regeneration with functional return of vision. Proceedings of the National Academy of Sciences 103: 5054-5059. 
27. Gurtner GC, Werner S, Barrandon Y, Longaker MT (2008) Wound repair and regeneration. Nature 453: 314-321. [Crossref]

28. Sun G, Zhang X, Shen YI, Sebastian R, Dickinson LE, et al. (2011) Dextran hydrogel scaffolds enhance angiogenic responses and promote complete skin regeneration during burn wound healing. Proceedings of the National Academy of Sciences 108: 2097620981.

29. Place ES, Evans ND, Stevens MM (2009) Complexity in biomaterials for tissue engineering. Nat Mater 8: 457-470. [Crossref]

30. Jayawarna V, Ali M, Jowitt TA, Miller AF, Saiani A, et al. (2006) Nanostructured hydrogels for three-dimensional cell culture through self-assembly of fluorenylmethoxycarbonyl-dipeptides. Adv Mater 18: 611-614.

31. Dunn CJ, Goa KL (1999) Tranexamic acid: a review of its use in surgery and other indications. Drugs 57: 1005-1032. [Crossref]

32. Martinez-Gutierrez F, Olive PL, Banuelos A, Orrantia E, Nino N, et al. (2010) Synthesis, characterization, and evaluation of antimicrobial and cytotoxic effect of silver and titanium nanoparticles. Nanomedicine 6: 681-688.

33. Lakshmanan A, Cheong DW, Accardo A, Di Fabrizio E, Riekel C, et al. (2013) Aliphatic peptides show similar self-assembly to amyloid core sequences, challenging the importance of aromatic interactions in amyloidosis. Proceedings of the National Academy of Sciences 110: 519-524.

34. Greenfield MA, Hoffman JR, de la Cruz MO, Stupp SI (2010) Tunable mechanics of peptide nanofiber gels. Langmuir 26: 3641-3647. [Crossref]

35. Mezger TG, The rheology handbook: for users of rotational and oscillatory rheometers, Vincentz Network GmbH \& Co KG 2006

36. Dominguez R, Holmes KC (2011) Actin structure and function. Annu Rev Biophys 40: 169-186. [Crossref]
37. Boucard N, Viton C, Agay D, Mari E, Roger T, et al. (2007) The use of physical hydrogels of chitosan for skin regeneration following third-degree burns. Biomaterials 28: 3478-3488.

38. Sullivan TP, Eaglstein WH, Davis SC, Mertz P (2001) The pig as a model for human wound healing. Wound Repair Regen 9: 66-76. [Crossref]

39. Velander P, Theopold C, Hirsch T, Bleiziffer O, Zuhaili B, et al. (2008) Impaired wound healing in an acute diabetic pig model and the effects of local hyperglycemia. Wound Repair Regen 16: 288-293.

40. Losi P, Briganti E, Errico C, Lisella A, Sanguinetti E, et al. Fibrin-based scaffold incorporating VEGF-and bFGF-loaded nanoparticles stimulates wound healing in diabetic mice, Acta Biomaterialia 9 (2013) 7814-7821

41. Yanagibayashi S, Kishimoto S, Ishihara M, Murakami K, Aoki H, et al. (2012) Nove hydrocolloid-sheet as wound dressing to stimulate healing-impaired wound healing in diabetic db/db mice. Biomed Mater Eng 22: 301-310.

42. Bielefeld KA, Amini-Nik S, Alman BA (2013) Cutaneous wound healing: recruiting developmental pathways for regeneration. Cell Mol Life Sci 70: 2059-2081.

43. Selig HF, Lumenta DB, Giretzlehner M, Jeschke MG, Upton D, et al. (2012) The properties of an "ideal" burn wound dressing-what do we need in daily clinical practice? Results of a worldwide online survey among burn care specialists. Burns 38: $960-966$.

44. Cameron P, Gaiser BK, Bhandari B, Bartley PM, Katzer F, et al. (2016) Silve nanoparticles decrease the viability of Cryptosporidium parvum oocysts. Appl Environ Microbiol 82: 431-437.

45. Carrejo NC, Moore AN, Lopez Silva TL, Leach DG, Li IC, et al. (2018) Multidomain Peptide Hydrogel Accelerates Healing of Full-Thickness Wounds in Diabetic Mice. ACS Biomater Sci Eng 4: 1386-1396.

Copyright: (C2018 Wafaa T Arab and Charlotte AE Hauser. This is an open-access article distributed under the terms of the Creative Commons Attribution License, which permits unrestricted use, distribution, and reproduction in any medium, provided the original author and source are credited. 